\title{
Corrigendum
}

\section{Correction to equation (5.6) in the paper: A queueing network with a single cyclically roving server}

\author{
Moshe Sidi \\ Electrical Engineering Department, Technion-Israel Institute of Technology, \\ Haifa 32000, Israel \\ Hanoch Levy \\ Computer Science Department, Tel-Aviv University, \\ Tel Aviv 69978, Israel \\ Steve W. Fuhrmann \\ 1 Independence Court, Morristown, NJ 07960, USA
}

Received 1 October 1993

R. Ram and A. Tripathi have pointed out that a term is missing in the expression given in [1] (eq (5.6)) for computing the expected path delay in a gated service system. The missing term is the contribution of the server switch-over times to the path delays. Equation (5.6) should therefore read:

$$
\begin{aligned}
E\left[T_{i_{1}, i_{2}, \ldots, i_{M}}\right]= & {\left[g_{i_{1}}\left(i_{1}\right)-\bar{g}_{i_{1}}\left(i_{1}\right)-1\right] b_{i_{1}}+\sum_{m=2}^{M-1} g_{i_{m}}\left(i_{m}\right) b_{i_{m}}+\left[\bar{g}_{i_{M}}+1\right] b_{i_{M}} } \\
& +\sum_{m=1}^{M-1} \sum_{n=1}^{k_{m}} g_{i_{m}+n}\left(i_{m}+n\right) b_{i_{m}+n}+\sum_{m=1}^{M-1}\left[r_{i_{m}}+\sum_{n=1}^{k_{m}} r_{i_{m}+n}\right]
\end{aligned}
$$

We would like to thank R. Ram and A. Tripathi for pointing out the missing term.

\section{Reference}

[1] M. Sidi, H. Levy and S.W. Fuhrmann, A queueing network with a single cyclically roving server, Queueing Syst. 11 (1992) 121-144. 